\title{
MicroRNA-374a promotes esophageal cancer cell proliferation via Axin2 suppression
}

\author{
YUE WANG, HUA XIN, ZHIFENG HAN, HONGBING SUN, NAN GAO and HAIXIANG YU \\ Department of Thoracic Surgery, China-Japan Union Hospital of Jilin University, Changchun, Jilin 130033, P.R. China
}

Received February 19, 2015; Accepted May 18, 2015

DOI: $10.3892 /$ or.2015.4182

\begin{abstract}
MicroRNA-374a (miR-374a) is involved in the progress of various types of cancer, and may indicate a poor prognosis. However, its role in esophageal cancer remains to be determined. In the present study, the role of miR-374a in esophageal cancers and cancer cell growth was examined using miR-374a overexpression and underexpression models. The results showed that miR-374a was markedly increased in esophageal cancer cell lines and tumor samples from patients with esophageal cancer. In esophageal cancer Eca109 cells, the ectopic overexpression of miR-374a promoted cell growth. Additionally, cell growth was reduced by miR-374a inhibition. The mechanisms underlying the promotive role were examined and it was found that miR-374a significantly decreased the expression and transcription activity of axis inhibition protein 2 (Axin2). Axin2, a tumor suppressor, exhibited a marked inhibitory effect on Eca109 cell growth. The results identified a new role of miR-374a in esophageal cancer involving Axin2 suppression.
\end{abstract}

\section{Introduction}

Esophageal cancer, especially squamous cell carcinoma, is considered as one of the most aggressive types of cancer with poor prognosis (1). Traditionally, surgery is considered the best treatment in the early stage. In advanced esophageal cancer, a multidisciplinary approach that includes surgery, radiotherapy, and chemotherapy, alone or in combination, has been developed (2). However, patient survival remains unsatisfactory.

In the last decade, the efforts with regard to cancer research in the field of non-coding microRNA (miRNA) have increased exponentially. miRNA-based therapeutics have been tested

Correspondence to: Dr Haixiang Yu, Department of Thoracic Surgery, China-Japan Union Hospital of Jilin University, No. 126 Xiantai Street, Changchun, Changchun, Jilin 130033, P.R. China E-mail: gerry422@163.com

Key words: Axin2, axis inhibition protein 2, miR-374a, esophageal cancer at the preclinical and clinical stages, and miRNA-based cancer diagnostics and prognostics have been applied clinically to manage cancer treatment (3). miRNAs interact with partially complementary sequences in the 3'-UTR of the target mRNAs and negatively modulate gene expression at the post-transcriptional level. The fine-tuning of the cells through miRNAs and other factors controls several central biological processes in many different species (4). Aberrant regulation of miRNAs has been involved in a number of human diseases, including cancers. In tumorigenesis, miRNAs may act as tumor suppressors $(5,6)$ or oncogenes $(7,8)$ in different types of cancers, including esophageal cancer (9). Recent clinical findings indicated that the upregulation of miR-146b was associated with poor survival in esophageal squamous cell carcinoma (10). miR-645 was upregulated in human adenocarcinoma of the gastric esophageal junction and inhibited apoptosis by targeting tumor suppressor IFIT2 (11). miR-100 promoted migration and invasion through mammalian target of rapamycin in esophageal squamous cell carcinoma (12). By contrast, miR-197 was downregulated in esophageal cancer with a poor prognosis, and may play a tumor-suppressor role (13). Additionally, miR-302b inhibited cell proliferation by inducing apoptosis and suppressing the invasion in esophageal squamous cell carcinoma (14).

miRNA profiling has shown that microRNA-374a (miR-374a) is overexpressed in many types of cancer, such as head and neck squamous cell carcinoma, follicular lymphoma, osteosarcoma and bladder urothelial carcinoma (15-18). In breast cancer, miR-374a was markedly upregulated in primary tumor samples from patients with distant metastases and was considered to be associated with poor metastasis-free survival (7). In gefitinib-resistant lung cancer cells, the expression of miR-374a was high (19). By contrast, in early-stage non-small cell lung cancer, the expression of miR-374a was low (4). However, the expression and clinical significance of miR-374a in esophageal cancer remains to be determined.

To determine the role of miR-374a in esophageal cancers, we introduced miR-374a overexpression and underexpression models in the present study. Using these models, we investigated the effect of miR-374a on esophageal cancer cell growth. Axis inhibition protein 2 (Axin2), a tumor suppressor present in a number of human cancers (20), has a complementary binding site of miR-374a at 3'-UTRs, which indicates Axin2 is a putatively potential target of miR-374a. Thus, the effect of miR-374a on Axin2 was explored. 


\section{Materials and methods}

Reagents. Fetal bovine serum (FBS), Lipofectamine 2000 reagent, non-essential amino acids, RPMI-1640 medium and TRIzol reagent were purchased from Life Technologies (Grand Island, NY, USA). Esophageal cancer cell lines, Eca109, Kyse30, Kyse140, Kyse180, Kyse410, Kyse510, Kyse520, and TE-1, as well as the normal esophageal epithelial cell (NEEC) line, were purchased from the American Type Culture Collection (ATCC; Manassas, VA, USA). 3-(4,5-Dimethylthiazol-2-yl)2,5-diphenyltetrazolium bromide (MTT), agar, propidium iodide, puromycin, and trypsin were purchased from SigmaAldrich (St. Louis, MO, USA). Antibodies against $\alpha$-tubulin, Axin2, cyclin $\mathrm{D} 1$, phosphorylated $\mathrm{Rb}$ or $\mathrm{Rb}$ were purchased from Abcam Ltd. (Cambridge, UK). Horseradish peroxidase-coupled secondary IgGs were purchased from Santa Cruz Biotechnology (Dallas, TX, USA). ECL reagent was purchased from Pierce (Rockford, IL, USA). The luciferase Reporter Assay system, $B g l \mathrm{II}, M l u \mathrm{I}$ and $\mathrm{XhoI}$ were purchased from Promega (Madison, WI, USA). pSuper-puro and pGL3-Basic Luciferase reporter plasmid were purchased from Clontech Laboratories, Inc. (Mountain View, CA, USA). miR-374a inhibitor and its negative control were purchased from Qiagen (Valencia, CA, USA).

Subjects. In total, 46 patients who received esophageal cancer surgery at the China-Japan Union Hospital of Jilin University between April, 2012 and April, 2014 were selected. The diagnosis of esophageal cancer was confirmed pathologically. These patients had not previously received chemotherapy or radiotherapy. Biopsies were obtained from the esophageal cancer tissues and normal tissues adjunct to cancer area during surgery. Tissues were used for pathology to confirm the diagnosis and for mRNA extraction and RT-PCR. All 46 patients provided written informed consent. The study protocol was approved by the China-Japan Union Hospital of Jilin University.

Cell culture. Esophageal cancer cell lines, Eca109, Kyse30, Kyse140, Kyse180, Kyse410, Kyse510, Kyse520, TE-1, and the NEEC line were grown in RPMI-1640 medium, supplemented with heat-inactivated FBS $(10 \% \mathrm{v} / \mathrm{v}), 100 \mathrm{U} / \mathrm{ml}$ penicillin, $100 \mu \mathrm{g} / \mathrm{ml}$ streptomycin and non-essential amino acids (1\% $\mathrm{v} / \mathrm{v}$ ) and cultured at $37^{\circ} \mathrm{C}$ in an atmosphere of $5 \% \mathrm{CO}_{2}$ with a relative humidity of $95 \%$.

Stable transfection of miR-374a in Ecal09 cells. The miR-374a expression plasmid was generated by cloning the pre-miR-374a gene with $B g l \mathrm{II}$ and $\mathrm{XhoI}$ endonuclease sites on each flanking side into retroviral transfer plasmid pSuper-puro to generate the pSuper-puro-miR-374a plasmid. A scrambled miRNA with no homology to any known human miRNAs was used as the negative control. These plasmids were transfected into Eca109 cells using Lipofectamine 2000 reagent according to the manufacturer's instructions. After $36 \mathrm{~h}$ of transfection, the cells were selected using selection medium containing $1.5 \mu \mathrm{g} / \mathrm{ml}$ puromycin. The surviving cells, which were considered as stably transfected cells, were maintained and passaged in culture medium containing puromycin.
Transient transfection of Axin2 siRNA and miR-374a inhibitor. The transfection of Axin2 siRNA, miR-374a inhibitor and their respective control oligonucleotides into Eca109 cells was performed using Lipofectamine 2000 reagent according to the manufacturer's instructions. The sequences of siRNA and its negative control were as follows: Axin2,5'-GCAGAGG GACAGGAATCAT-3', and the negative control, 5'-GCAGGG ACAAGGTAGACAT-3'.

Luciferase assay. The Axin23'-UTR was amplified from human genomic DNA and subcloned into the pGL3-basic luciferase reporter plasmid using $M l u \mathrm{I}$ and $\mathrm{XhoI}$ sites. Axin2 3'-UTR luciferase reporter plasmids were transfected into Eca109 cells that stably expressed miR-374a using Lipofectamine 2000 reagent. Axin2 3'-UTR luciferase reporter plasmids were also co-transfected with miR-374a inhibitor into intact Eca109 cells using Lipofectamine 2000 reagent. Transfected cells were serum-shocked (20\%) for $2 \mathrm{~h}$ and maintained in $2 \%$ media for $24 \mathrm{~h}$ prior to cell collection. The dual-luciferase reporter assay was performed according to the manufacturer's instructions. Data were presented as relative luciferase activity.

Cell proliferation assay. Cell proliferation was analyzed with MTT as previously described (21). Briefly, $1 \times 10^{3}$ cells of each group were plated in 96-well microplates in $150 \mu \mathrm{l}$ of medium. After different time of culture, $20 \mu 1$ of MTT substrate $(5 \mathrm{mg} /$ $\mathrm{ml}$ ) was added to each well, and the plates were incubated for an additional $4 \mathrm{~h}$. The medium was then removed, and the cells were solubilized in $150 \mu \mathrm{l}$ of dimethyl sulfoxide. The culture plates were then agitated for $15 \mathrm{~min}$ and the optical absorbance values were measured at $490 \mathrm{~nm}$.

Colony formation assay. Following the treatments, the cells were trypsinized and counted. Cells $\left(1 \times 10^{3}\right)$ of each group were seeded in 12 -well plates. The number of colonies was counted at the 5th day after seeding. The trypsinized cells were also seeded for an anchorage-independent growth ability assay as previously described (22). One thousand cells were suspended in $2 \mathrm{ml}$ complete medium plus $0.33 \%$ agar. The agar-cell mixture was plated on top of a bottom layer consisting of $0.66 \%$ complete medium agar mixture. After 5 days, the colony sizes were measured using an ocular micrometer and colonies $>0.1 \mathrm{~mm}$ in diameter were counted.

Flow cytometric analysis. Flow cytometric analysis was performed as previously described (22). The cells were harvested by trypsinization, washed in ice-cold PBS, and fixed in $80 \%$ ice-cold ethanol. Prior to staining, the cells were pelleted in a cooled centrifuge and resuspended in cold PBS. The cells were then incubated in $20 \mu \mathrm{g} / \mathrm{ml}$ propidium iodide for $20 \mathrm{~min}$ at room temperature, and analyzed on a flow cytometer (FACSCalibur; BD Biosciences, Bedford, MA, USA).

RNA extraction and RT-PCR. Total RNA was extracted from human tissues and cells using TRIzol reagent, and purified using a miRNeasy column (Qiagen). The RNA was reverse transcribed into cDNA using a Reverse Transcript reagent kit. miR-374a expression was determined using TaqMan microRNA assays (Applied Biosystems, Grand Island, NY, USA). U6, a small nuclear RNA, was used as an internal 

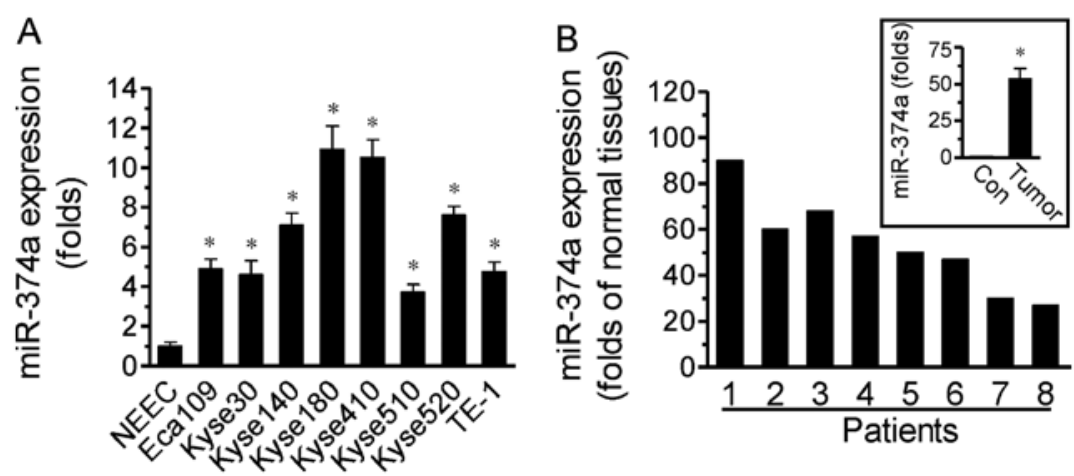

Figure 1. miR-374a expression is increased in esophageal cancer cell lines and tissues. (A) mRNA expression of miR-374a was measured with real-time PCR in esophageal cancer cell lines and the normal esophageal epithelial cells (NEEC). (B) The expression of miR-374a in 46 paired cancer tissues (tumor) and their adjacent non-cancerous esophageal tissues (Con). The figure is representative of 8 patients, and the miR-374a expression in cancer tissues was presented as the folds of respective non-cancerous esophageal tissues. The insert is for all 46 patients. ${ }^{*} \mathrm{P}<0.01$ vs. NEEC or Con.

control. Specific TaqMan primers, targeting mature miR-374a or U6 were obtained from Applied Biosystems. RT-PCR was performed in an ABI 7500 Real-Time PCR system. The results were presented as the relative changes of miR-374a following normalization to U6.

Western blot analysis. The protein levels of Axin2, cyclin D1 and $\mathrm{p}-\mathrm{Rb}$ were assessed using western blotting as previously described (23). Whole-cell proteins were separated electrophoretically in $4-12 \%$ SDS-PAGE gels, and transferred to nitrocellulose membranes. Following 30 min of blocking with $2.5 \%$ non-fat milk, the membranes were incubated with primary antibody (dilution at 1:1,000 1:2,000) at $4^{\circ} \mathrm{C}$ overnight, and followed by 1 -h incubation with horseradish peroxidase-conjugated secondary antibody (1:2,000). The membranes were adequately washed with PBS containing $0.5 \%$ Tween-20 subsequent to each treatment with antibody. The membranes were developed with chemiluminescence reagent and then exposed to X-ray film. The protein levels were presented as the ratio of the band optical intensity to that of $\alpha$-tubulin or total $\mathrm{Rb}$.

Statistical analysis. Data are presented as mean \pm SEM. Statistical analysis was performed using one-way analysis of variance, followed by Dunnett's test for multiple comparisons and by the Student's t-test for comparisons between two groups. $\mathrm{P}<0.05$ was considered to indicate statistical difference.

\section{Results}

miR-374a expression is increased in various esophageal cancer cell lines and human esophageal cancer tissues. We first examined the miR-374a levels in various esophageal cancer cells using real-time PCR. The results showed that miR-374a levels were significantly increased in eight types of esophageal cancer cells, Eca109, Kyse30, Kyse140, Kyse180, Kyse410, Kyse510, Kyse520 and TE-1, as compared to the NEEC line $(\mathrm{P}<0.01$, Fig. 1A). We further examined whether the increased miR-374a level existed in human esophageal cancer tissues. The mRNA was extracted from cancer and adjunct normal esophageal tissues from 46 patients with esophageal cancer, and real-time PCR was performed to measure miR-374a expression. When compared to the normal esophageal tissues, miR-374a levels in cancer tissues were increased in each pair of samples, and were significantly higher collectively $(\mathrm{P}<0.01$, Fig. 1B). These results demonstrated that miR-374a expression is increased in esophageal cancer, and suggested that miR-374a is associated with the development of esophageal cancer.

Ectopic overexpression of miR-374a promotes cell proliferation. To examine the role of the increased miR-374a in esophageal cancer, esophageal cancer Eca109 cells were transfected with miR-374a-containing vector or scrambled miRNA. The stable overexpression of miR-374a was obtained after antibiotic selection, and was confirmed by RT-PCR (Fig. 2A). The same amount of Eca109 cells, which stably overexpressed miR-374a or scrambled control, were seeded to examine the effect of miR-274a on cell proliferation. In hte MTT assay, miR-374a overexpression yielded a more rapid increase in the MTT absorbance value with culture time, compared to scramble control (Fig. 2B). In addition, miR-374a overexpression increased the relative colony number (Fig. 2C) and the colony number with size $>0.1 \mathrm{~mm}$ (Fig. 2D). When analyzing the cell cycle using flow cytometry, we found that ectopic overexpression of miR-374a induced a significant decrease in the percentage of cells in the G1/G0 phase and an increase in the percentage of cells in the $\mathrm{S}$ phase (Fig. 2E). These results suggested that the upregulation of miR-374a promotes the Eca109 cell proliferation.

Underexpression of miR -374 a reduces cells proliferation. We introduced an underexpression model of miR-374a to further validate the promotive role of miR-374a in esophageal cancer cells proliferation. An antisense-based specific inhibitor against miR-374a was applied to inhibit the expression of miR-374a in Eca109 cells (Fig. 3A). Unlike miR-374a overexpression, miR-374a inhibition significantly decreased the MTT absorbance value with culture time, as compared to its negative control oligonucleotides (Fig. 3B). miR-374a inhibition also reduced cell colony formation (Fig. 3C and D). Additionally, flow cytometry showed a significant increase in the percentage of cells in the G1/G0 phase and a decrease in the percentage of cells in the S phase by miR-374a inhibitor, as compared to its negative control oligonucleotides (Fig. 3E). 
A
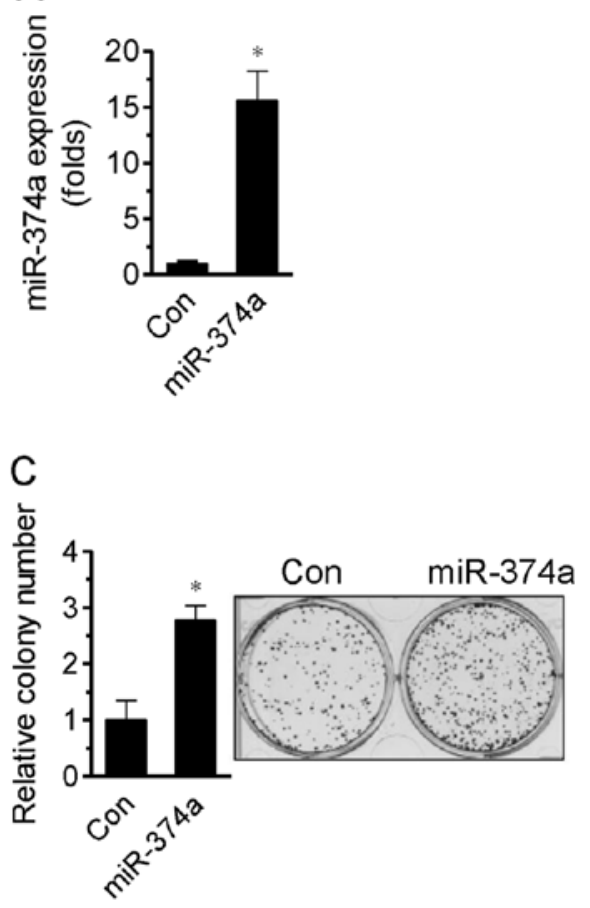

$\mathrm{B}$

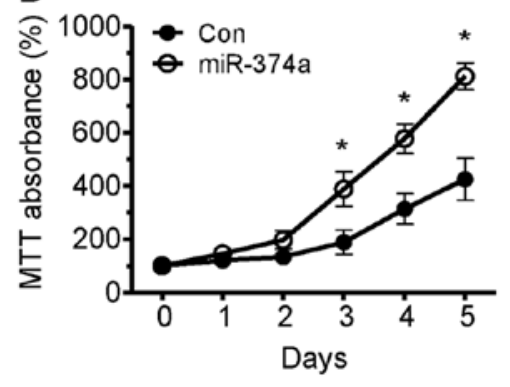

D

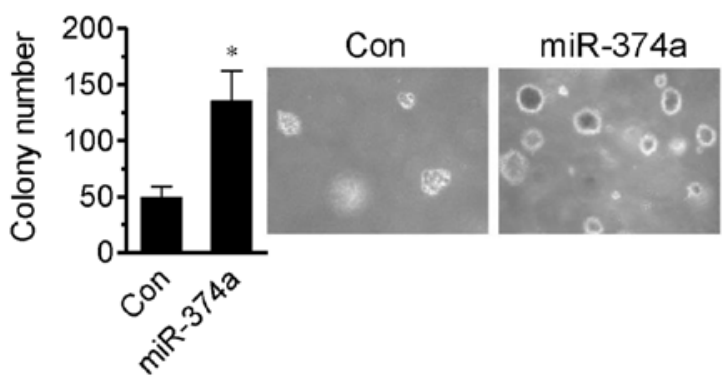

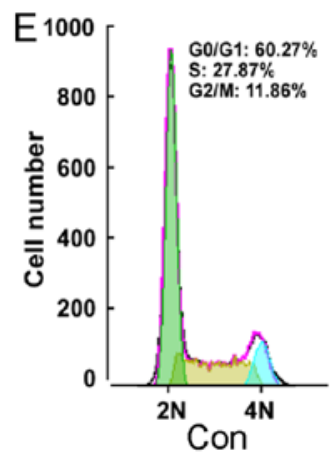

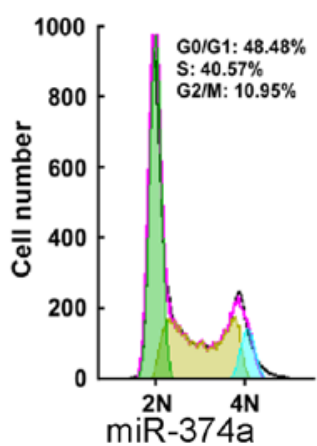

Figure 2. Ectopic overexpression of miR-374a promotes Eca109 cell proliferation. (A) mRNA expression of miR-374a was measured with real-time PCR analysis in Eca109 cells stably expressing miR-374a or scrambled control (Con). (B) Effects of miR-374a overexpression on the cells proliferation were analyzed by an MTT assay. (C) The number of colonies in cells with miR-374a overexpression or control cells was counted 5 days after seeding. Representative micrographs (right) and quantification (left) are shown. (D) Cell colonies from an anchorage-independent growth ability assay. Colonies $>0.1 \mathrm{~mm}$ in diameter were counted. (E) Effects of miR-374a overexpression on the cell cycle were measured using flow cytometric analysis. "P<0.05 vs. Con, or day 0 .

These results suggested that the underexpression of miR-374a suppressed the proliferation of Eca109 cells.

miR-374a represses Axin2 expression. The 3'-UTRs of Axin2 have a binding site of miR-374a, which indicates Axin2 is a putative potential target of miR-374a (Fig. 4A). To confirm the relationship of miR-374a with Axin2, a luciferase reporter plasmid containing Axin2 3'-UTR was transfected into miR-374a-overexpressed Eca109 cells. In the dual-luciferase assay, miR-374a overexpression significantly decreased luciferase activity, as compared to miR-374a-scrambled Eca109 cells. When the luciferase reporter plasmids were transfected into Eca109 cells with miR-374 inhibitor, miR-374 inhibitor control oligonucleotides or miR-374a mutant, the luciferase activity was significantly increased by miR-374 inhibitor, compared to the control oligonucleotides, while the miR374a mutant did not change the luciferase activity (Fig. 4B).
Thus, these results demonstrated that miR-374a suppressed Axin2 transcription activity. Following the Axin 2 protein analysis using western blotting, miR-374a overexpression was decreased, whereas miR-374a inhibition increased the Axin2 protein level (Fig. 4C). In addition, miR-374a exhibited a promotive effect on the tumor-related factors, cyclin D1 and Rb. miR-374a overexpression increased cyclin D1 expression and $\mathrm{Rb}$ phosphorylation, while miR-374a inhibition decreased cyclin D1 expression and Rb phosphorylation. miR-374a overexpression and miR-374a inhibition did not affect the levels of total $\mathrm{Rb}$ (Fig. 4D).

Axin2 knockdown increases Ecal09 cell proliferation. Axin2 is a negative regulator of the canonical Wnt/ $\beta$-catenin signaling pathway, and acts as a tumor-suppressor protein in a number of human cancers (24). However, it is less involved in the role of Axin2 in esophageal cancer. To examine 


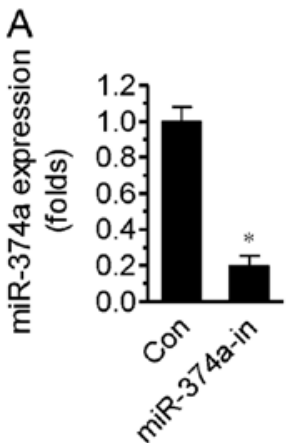

C

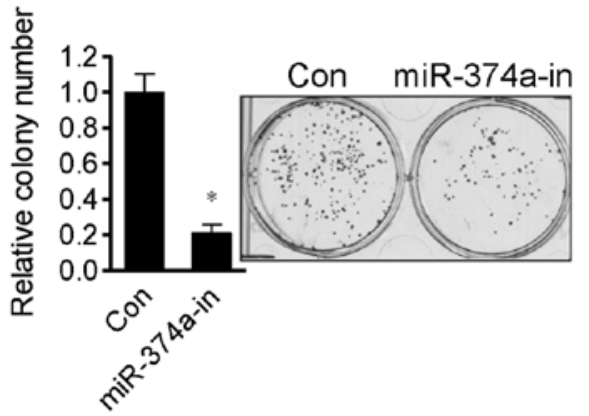

$\mathrm{B}$

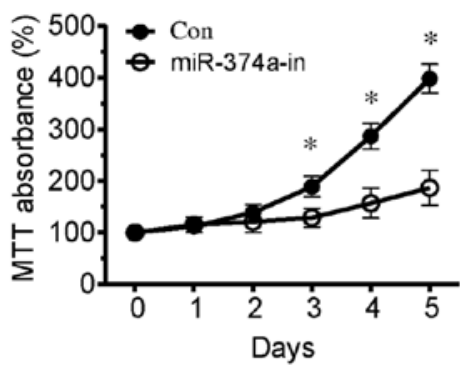

$\mathrm{D}$

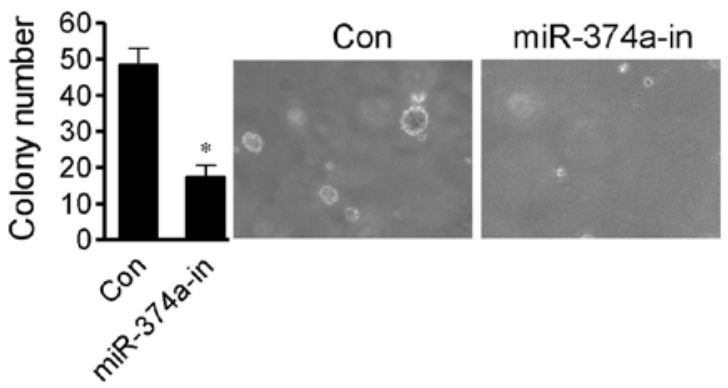

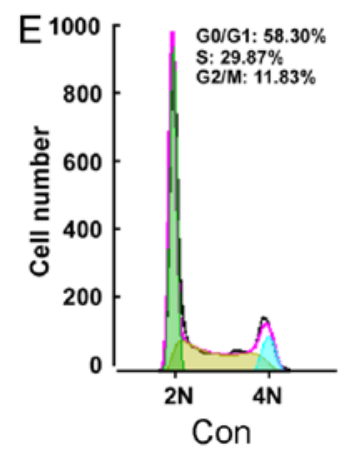

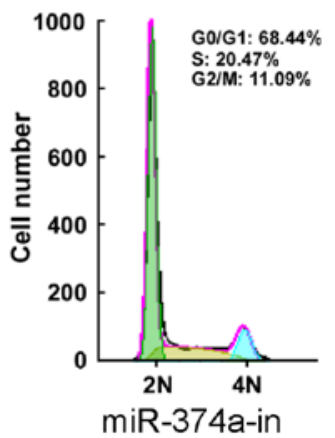

Figure 3. Underexpression of miR-374a reduces Eca109 cell proliferation. (A) mRNA expression of miR-374a was measured in Eca109 cells transfected with miR374a inhibitor (miR-374a in) or negative control (Con). (B) Effects of miR-374a inhibition on cell proliferation as analyzed by MTT assay. (C) The number of cells colonies was counted 5 days after the inoculation of miR-374a inhibitor or negative control. (D) Cell colonies from the anchorage-independent growth ability assay. Colonies $>0.1 \mathrm{~mm}$ in diameter were counted. (E) Effects of miR-374a inhibition on the cell cycle were measured using flow cytometry. ${ }^{*} \mathrm{P}<0.05$ vs. Con, or day 0 .

whether decreased Axin2 plays a tumor-suppressor role in esophageal cancer, we applied specific Axin2 siRNA to silence Axin 2 in Eca109 cells, which were simultaneously treated by the miR-374a inhibitor (Fig. 5A). In the colony formation assay, Axin2 siRNA significantly increased the relative colony number (Fig. $5 \mathrm{~B}$ ) and colony number with a size of $>0.1 \mathrm{~mm}$ (Fig. 5C), compared to the negative control of Axin2 siRNA. These results suggested that Axin2 may exert a suppressive effect on Eca109 cell proliferation.

\section{Discussion}

The aim of the present study was to assess the role of miR-374a in the regulation of proliferation of esophageal cancer cells and the possible mechanisms. We found that miR-374a expression was significantly increased in various esophageal cancer cell lines and cancer tissues. miR-374a overexpression significantly increased esophageal cancer cell proliferation, whereas miR-374a underexpression decreased the cell proliferation.
The overexpression and underexpression of miR-374a exhibited a decreased and an increased effect on the transcription activity as well as the expression of Axin2, a tumor suppressor, respectively. Axin2, in a siRNA-mediated knockdown model, revealed a tumor-suppressive effect on esophageal cancer cells. Thus, our results demonstrate a promotive role of miR-374a in esophageal cancer, which may be mediated by the suppression of Axin2.

miRNAs have been shown to be a significant gene modulator during esophageal cancer progression, and global miRNA expression profiles have been performed using microarray, RT-PCR or sequencing approaches (10,25-28). In the present study, we used RT-PCR to identify the increased expression of miR-374a in esophageal cancer cell lines and cancer tissues. miR-374a has diverse biological functions in different tumor types. miR-374a even exhibits contrast regulation in different cancer types, including upregulation in most cancer types $(7,15-18)$ and downregulation in earlystage non-small cell lung cancer (4). miR-374a was markedly 
A

$\begin{aligned} & \text { Axin2 3'-UTR 5'-1306 UACUGUAAAUUAUGCAUUAUAAA1328-3' } \\ & \text { miR-374a } \\ & \text { 3'-GUGAAUAGUCCAACAUAAUAUU-5' } \\ & \text { miR-374a-mutant } \\ & \text { 3'-GUGAAUAGUCCAACAUAGCGUU-5' }\end{aligned}$

C

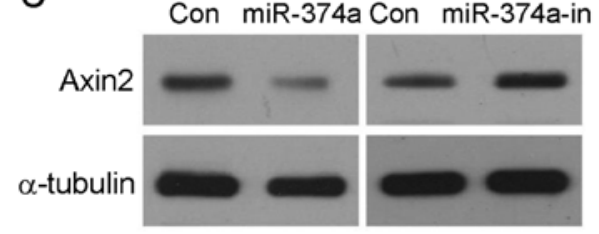

$\mathrm{B}$
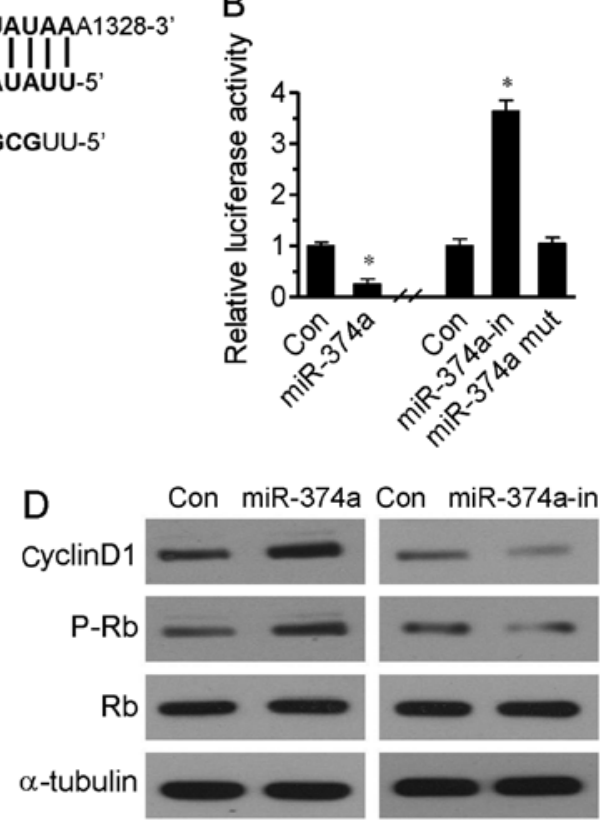

Figure 4. miR-374a represses Axin2 expression. (A) Schematic miR-374a putative target sites in 3'-UTR of Axin2, and a miR-374a mutant containing three altered nucleotides in the core sequence (miR-374a-mut). (B) Luciferase assay of indicated cells transfected with the Axin2 3'-UTR reporter. (C) The expressions of Axin2 protein was measured by western blotting in Eca109 cells stably overexpressing miR-374a, or transiently suppressing miR-374a (miR-374a-in, $48 \mathrm{~h}$ ) or their respective control (Con). (D) Expression of cyclin D1, Rb and phosphorylated Rb (P-Rb) protein was measured by western blotting in the indicated Eca109 cells. ${ }^{*} \mathrm{P}<0.05$ vs. Con. Axin2, axis inhibition protein 2.

A

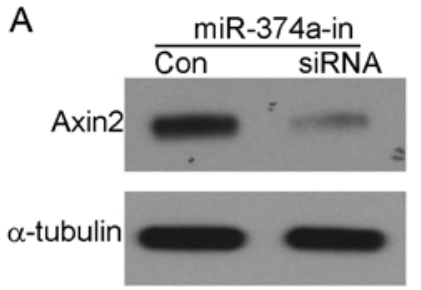

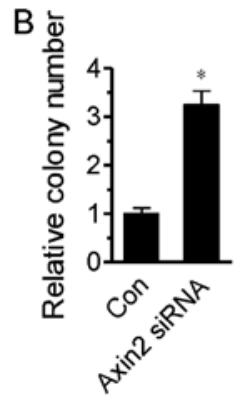

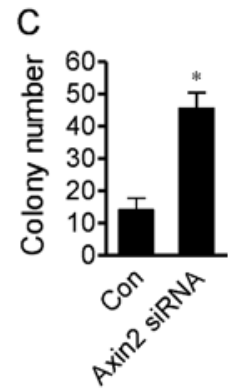

Figure 5. Axin2 knockdown increases cell proliferation. (A) The expression of Axin2 in Eca109 cells transfected with Axin2 siRNA and miR-374a inhibitor (miR-374a-in) was measured by western blotting. (B) The number of cell colonies in the indicated Eca109 cells was counted 5 days after inoculation. (C) Colony numbers from an anchorage-independent growth assay. Colonies $>0.1 \mathrm{~mm}$ in diameter were counted. ${ }^{*} \mathrm{P}<0.05 \mathrm{vs}$. Con.

upregulated in the primary breast cancer tumor samples, and the upregulation promoted epithelial-mesenchymal transition and metastasis in vitro and in vivo (7), suggesting an important role of miR-374a in breast cancer tumorigenesis. Consistent with those studies, in the present study, the overexpression of miR-374a exhibited a promotive effect on the proliferation of esophageal cancer cells. Additionally, when miR-374a expression was downregulated by its inhibitor, cell proliferation was significantly decreased. Thus, these results, together with the high expression in esophageal cancer tissue, demonstrate the important role of miR-374a in esophageal cancer development.

miRNAs exert their function by interacting with the 3'-UTR of the target mRNAs. miR-374a was reported to directly target and suppress multiple negative regulators of the Wnt/ $\beta$-catenin signaling cascade (7). As a member of $\beta$-catenin/T-cell factor-dependent genes, Axin2 has complementary sequences of the miR-374a core region (Fig. 4A), which indicates a possible interaction between miR-374a and Axin2. When co-infected with luciferase reporter plasmids containing Axin2 3'-UTR, miR-374a markedly decreased the luciferase activity. This finding suggests that miR-374a decreases Axin 2 transcription. By contrast, miR-374a inhibition markedly increased luciferase activity, providing further support for the suppressive effect of miR-374a on Axin2 transcription. When considering whether the transcription inhibition was transferred to the protein expression, our results showed the Axin2 protein level was decreased by miR-374a overexpression, but increased by miR-374a inhibition. These results demonstrate that miR-374a is an effective inhibitor of Axin2 expression.

$\mathrm{Wnt} / \beta$-catenin signaling is important in the development of a broad range of human cancers (29). However, investigation of the Wnt pathway components in esophageal squamous cell carcinoma is less involved. In previous studies, the expression 
of Axin was reduced in almost $46 \%$ of investigated esophageal squamous cell carcinoma specimens, and was inversely correlated with tumor invasion depth and progression (30,31). In the present study, we knocked down Axin2 using Axin2-specific siRNA. The knockdown of Axin2 significantly increased the proliferation of Eca109 cells, indicating the suppressive role of Axin 2 in esophageal cancer. Thus, Axin 2 suppression by miR374a may stress the mechanism by which miR-374a promotes esophageal cancer growth.

In addition, miR-374a was also found to increase the levels of cyclin D1 and Rb phosphorylation, two cancer-related factors. Cyclin D1 was inversely associated with Axin level in the lung cancer cell line (32), and $\beta$-catenin regulated the expression of cyclin D1 in colon carcinoma cells (33). Cyclin D1 expression and phosphorylation of $\mathrm{Rb}$ were also increased by another miRNA, miR-208, which promoted cell proliferation when ectopically expressed in esophageal squamous cell carcinoma lines (22). Thus, the increased expression of cyclin D1 and phosphorylation of Rb by miR374a provided a further mechanism for the tumor-promotive effect of miR-374a.

\section{References}

1. Enzinger PC and Mayer RJ: Esophageal cancer. N Engl J Med 349: 2241-2252, 2003

2. Yamasaki M, Miyata H, Miyazaki Y, Takahashi T, Kurokawa Y, Nakajima K, Takiguchi S, Mori M and Doki Y: Perioperative therapy for esophageal cancer. Gen Thorac Cardiovasc Surg 62: 531-540, 2014.

3. Fesler A, Zhai H and Ju J: miR-129 as a novel therapeutic target and biomarker in gastrointestinal cancer. Onco Targets Ther 7: 1481-1485, 2014

4. Võsa U, Vooder T, Kolde R, Fischer K, Välk K, Tõnisson N, Roosipuu R, Vilo J, Metspalu A and Annilo T: Identification of miR-374a as a prognostic marker for survival in patients with early-stage nonsmall cell lung cancer. Genes Chromosomes Cancer 50: 812-822, 2011.

5. Chen X, Gong J,Zeng H, Chen N, Huang R, Huang Y, Nie L, Xu M, Xia J, Zhao F, et al: MicroRNA145 targets BNIP3 and suppresses prostate cancer progression. Cancer Res 70: 2728-2738, 2010.

6. Wu D, Pan H, Zhou Y, Zhou J, Fan Y and Qu P: microRNA-133b downregulation and inhibition of cell proliferation, migration and invasion by targeting matrix metallopeptidase-9 in renal cell carcinoma. Mol Med Rep 9: 2491-2498, 2014.

7. Cai J, Guan H, Fang L, Yang Y, Zhu X, Yuan J, Wu J and Li M: MicroRNA-374a activates Wnt/ $\beta$-catenin signaling to promote breast cancer metastasis. J Clin Invest 123: 566-579, 2013.

8. Pineau P, Volinia S, McJunkin K, Marchio A, Battiston C, Terris B, Mazzaferro V, Lowe SW, Croce CM and Dejean A: miR-221 overexpression contributes to liver tumorigenesis. Proc Natl Acad Sci USA 107: 264-269, 2010.

9. Huang J, Zhang SY, Gao YM, Liu YF, Liu YB, Zhao ZG and Yang K: MicroRNAs as oncogenes or tumour suppressors in oesophageal cancer: Potential biomarkers and therapeutic targets. Cell Prolif 47: 277-286, 2014.

10. Li J, Shan F, Xiong G, Wang JM, Wang WL, Xu X and Bai Y: Transcriptional regulation of miR-146b by C/EBP $\beta$ LAP2 in esophageal cancer cells. Biochem Biophys Res Commun 446: 267-271, 2014

11. Feng X, Wang Y, Ma Z, Yang R, Liang S, Zhang M, Song S, Li S, Liu G, Fan D, et al: MicroRNA-645, up-regulated in human adencarcinoma of gastric esophageal junction, inhibits apoptosis by targeting tumor suppressor IFIT2. BMC Cancer 14: 633, 2014.

12. Zhang N, Fu H, Song L, Ding Y, Wang X, Zhao C, Zhao Y, Jiao F and Zhao Y: MicroRNA-100 promotes migration and invasion through mammalian target of rapamycin in esophageal squamous cell carcinoma. Oncol Rep 32: 1409-1418, 2014.

13. Wang TY, Liu SG, Zhao BS, Qi B, Qin XG and Yao WJ: Implications of microRNA-197 downregulated expression in esophageal cancer with poor prognosis. Genet Mol Res 13: $5574-5581,2014$
14. Zhang M, Yang Q, Zhang L, Zhou S, Ye W, Yao Q, Li Z, Huang C, Wen Q and Wang J: miR-302b is a potential molecular marker of esophageal squamous cell carcinoma and functions as a tumor suppressor by targeting ErbB4. J Exp Clin Cancer Res 33: 10, 2014.

15. Huang Y, Chuang A, Hao H, Talbot C, Sen T, Trink B, Sidransky D and Ratovitski E: Phospho- $\Delta \mathrm{Np} 63 \alpha$ is a key regulator of the cisplatin-induced microRNAome in cancer cells. Cell Death Differ 18: 1220-1230, 2011.

16. Wang W, Corrigan-Cummins M, Hudson J, MaricI, Simakova O, Neelapu SS, Kwak LW, Janik JE, Gause B, Jaffe ES, et al: MicroRNA profiling of follicular lymphoma identifies microRNAs related to cell proliferation and tumor response. Haematologica 97: 586-594, 2012.

17. Namløs HM, Meza-Zepeda LA, Barøy T, Østensen IH, Kresse SH, Kuijjer ML, Serra M, Bürger H, Cleton-Jansen AM and Myklebost O: Modulation of the osteosarcoma expression phenotype by microRNAs. PLoS One 7: e48086, 2012.

18. Zhang ZC, Huang Y, Wang XJ, Wang M and Ma LL: Expression of circulating microRNAs in patients with bladder urothelial carcinoma. Beijing Da Xue Xue Bao 45: 532-536, 2013 (In Chinese).

19. Wang Y, Xia H,Zhuang Z, Miao L, Chen X and Cai H: Axl-altered microRNAs regulate tumorigenicity and gefitinib resistance in lung cancer. Cell Death Dis 5: e1227, 2014.

20. Liu D, Li L, Yang Y, Liu W and Wu J: The Axin2 rs2240308 polymorphism and susceptibility to lung cancer in a Chinese population. Tumour Biol 35: 10987-10991, 2014.

21. Hu J, Fang Y, Cao Y, Qin R and Chen Q: miR-449a regulates proliferation and chemosensitivity to cisplatin by targeting cyclin D1 and BCL2 in SGC7901 cells. Dig Dis Sci 59: 336-345, 2014.

22. Li H, Zheng D, Zhang B, Liu L, Ou J, Chen W, Xiong S, Gu Y and Yang J: mir-208 promotes cell proliferation by repressing SOX6 expression in human esophageal squamous cell carcinoma. J Transl Med 12: 196, 2014.

23. Wang L, Wang J, Wang Y, Fu Q, Lei YH, Nie ZY, Qiu J and Bao TY: Protective effect of exogenous matrix metalloproteinase-9 on chronic renal failure. Exp Ther Med 7: 329-334, 2014.

24. Ma C, Liu C, Huang P, Kaku H, Chen J, Guo K, Ueki H, Sakai A, Nasu Y, Kumon H, et al: Significant association between the Axin2 rs2240308 single nucleotide polymorphism and the incidence of prostate cancer. Oncol Lett 8: 789-794, 2014.

25. Feber A, Xi L, Luketich JD, Pennathur A, Landreneau RJ, Wu M, Swanson SJ, Godfrey TE and Litle VR: MicroRNA expression profiles of esophageal cancer. J Thorac Cardiovasc Surg 135: 255-260, 2008.

26. Kimura S, Naganuma S, Susuki D, Hirono Y, Yamaguchi A, Fujieda S, Sano K and Itoh H: Expression of microRNAs in squamous cell carcinoma of human head and neck and the esophagus: miR-205 and miR-21 are specific markers for HNSCC and ESCC. Oncol Rep 23: 1625-1633, 2010.

27. Matsushima K, Isomoto H, Kohno S and Nakao K: MicroRNAs and esophageal squamous cell carcinoma. Digestion 82: 138-144, 2010.

28. Zhang C, Wang C, Chen X, Yang C, Li K, Wang J, Dai J, Hu Z, Zhou X, Chen L, et al: Expression profile of microRNAs in serum: A fingerprint for esophageal squamous cell carcinoma. Clin Chem 56: 1871-1879, 2010.

29. Park S, Yun E, Hwang IH, Yoon S, Kim DE, Kim JS, Na M, Song GY and Oh S: Ilimaquinone and ethylsmenoquinone, marine sponge metabolites, suppress the proliferation of multiple myeloma cells by down-regulating the level of $\beta$-catenin. Mar Drugs 12: 3231-3244, 2014.

30. Li AF, Hsu PK, Tzao C, Wang YC, Hung IC, Huang MH and Hsu HS: Reduced axin protein expression is associated with a poor prognosis in patients with squamous cell carcinoma of esophagus. Ann Surg Oncol 16: 2486-2493, 2009.

31. Nakajima M, Fukuchi M, Miyazaki T, Masuda N, Kato H and Kuwano H: Reduced expression of Axin correlates with tumour progression of oesophageal squamous cell carcinoma. Br J Cancer 88: 1734-1739, 2003.

32. Gao Y, Song C, Hui L, Li CY, Wang J, Tian Y, Han X, Chen Y, Tian DL, Qiu X, et al: Overexpression of RNF146 in non-small cell lung cancer enhances proliferation and invasion of tumors through the Wnt $/ \beta$-catenin signaling pathway. PLoS One 9: e85377, 2014.

33. Tetsu O and McCormick F: Beta-catenin regulates expression of cyclin D1 in colon carcinoma cells. Nature 398: 422-426, 1999. 